\title{
Impact De La Distribution Spatiale Des Stations-Service Dans \\ L'ecosysteme Urbain De Lubumbashi En Republique Democratique Du Congo
}

\author{
Sangwa Kiteba Guellord \\ Musisilwa Lwindi Elias \\ Nsenga Ilunga Jéremie \\ Asumani Salimini \\ UFR Biosciences
}

Département de Géographie et sciences de l'environnement, Université de Lubumbashi, Lubumbashi, RD Congo

Doi:10.19044/esj.2021.v17n7p211

Submitted: 27 June 2019

Accepted: 23 February 2021

Published: 28 February 2021
Copyright 2021 Author(s)

Under Creative Commons BY-NC-ND

4.0 OPEN ACCESS

Cite As:

Guellord S.K., Elias M.L., Jéremie N.I. \& Salimini A. (2021). Impact De La Distribution Spatiale Des Stations-Service Dans L'ecosysteme Urbain De Lubumbashi En Republique Democratique Du Congo. European Scientific Journal, ESJ, 17(7), 211.

https://doi.org/10.19044/esj.2021.v17n7p211

\section{Résumé}

Cet article se propose d'analyser les facteurs de localisation des stations-service et leurs impacts dans l'écosystème urbain de Lubumbashi. Pour y arriver, la méthode descriptive-explicative a été adoptée. À l'aide d'un Système de Positionnement Global (GPS), les stations-service ont été localisées. La méthode des quadrats a permis de mettre en évidence le modèle d'organisation spatiale de la ville et le logiciel Quantum Geographic Information System (QGIS) a rendu possible l'élaboration de la carte des stations-service. Après analyse, l'article ressort ce qui suit : les lieux d'activités intenses (centre des affaires), les grands axes et les carrefours attirent plus les opérateurs pétroliers qui cherchent à implanter les stationsservice au sein de la ville. Le prix abordable, la qualité du produit pétrolier, la proximité de la route, la rapidité de livraison de produits pétroliers attirent les clients vers les stations-service. L'impact environnemental de ces dernières a été élucidé et les risques liés à leur implantation sont grands car elles n'ont pas de zone tampon et leur rapprochement est un danger permanent d'incendie 
dans une ville où les véhicules anti incendies sont presque inexistants. D'où la mairie a une responsabilité pour la régulation des points de ventes des produits pétroliers afin de sauver des vies humaines.

Mots clés : Stations-Service, Opérateurs Pétroliers, Modèle Spatiale, Grands Axes, Carrefours, Impact Environnemental, Écosystème Urbain

\title{
Impact of the Spatial Distribution of Service Stations in the Urban Ecosystem of Lubumbashi in the Democratic Republic of Congo
}

\section{Sangwa Kiteba Guellord \\ Musisilwa Lwindi Elias \\ Nsenga Ilunga Jéremie \\ Asumani Salimini}

Department of Geography and environmental sciences,

University of Lubumbashi, Lubumbashi, Democratic Republic of Congo

\begin{abstract}
This paper focuses on analyzing the factors of the location of service stations and their impact in the spatial organization of the city of Lubumbashi. We have adopted the descriptive-explanatory method. The service stations were located using GPS. The quadrat method allowed us to highlight the spatial model of the city and the Quantum Geographic Information System (QGIS) software enabled us to develop the service station map. After the analysis of the quadrat and the map, we deduced the following: intense places of activity (business center) on the major roads and intersections which attracted more petroleum operators to establish service stations within the city. Affordable price, the quality of the petroleum product, proximity to the road, and the speed of delivery of petroleum products attracts customers to service stations. Their environmental impacts have been elucidated and the risks associated with their radiation are great because they have no buffer zones and their reconciliation is a permanent fire hazard in a city where fire fighting vehicles are almost nonexistent. Here, the town hall has a responsibility for regulating points of sales of petroleum products in order to save human lives.
\end{abstract}

Keywords: Service-Stations, Oil Operators, Spatial Model, Major Roads, Junctions, Environmental Impacts, Ecosystem Urban 


\section{Introduction}

Le développement de l'automobile a fait apparaître un nouveau type de spécialité dans la vente du carburant : les stations-service. Dès le début du siècle dernier, la station-service prend rapidement place dans le paysage et devient un lieu de la quotidienneté où se côtoient les personnes intéressées par le marché du carburant et du lubrifiant. Cependant, une telle installation, aujourd'hui banalisée, représente un risque de par le stockage et la distribution de liquides inflammables.

Pour gérer et prévenir les risques, il est nécessaire de les identifier, les localiser et les intégrer au sein de leur environnement. Cet article présente, à travers l'étude du réseau de distribution de carburant dans la Communauté Urbaine de Lubumbashi, les facteurs de répartition spatiale des stationsservice et leur influence sur l'écosystème urbain.

Le rôle du géographe ne pourra pas être négligé ; il lui revient la réflexion sur le modèle d'organisation spatiale des phénomènes. En rapport avec cette thématique, plusieurs études ont été menées, entre autres, celle de Cauvin et Rimbert (1976) qui a traité, à partir des méthodes cartographiques, des effets de localisation des stations-service sur l'environnement; celle de Blaesius (1992) sur les dernières nouvelles d'Alsace, et qui a abordé les stations hors services à partir de l'inventaire communal de 1988. Cette étude a révélé que les stations-service disparaissaient de plus en plus. Calvat (1988) quant à lui, s'intéressant à Paris, a trouvé que l'impact sur l'environnement est banalisé par nos sociétés actuelles. Heigel (1995) à son tour s'est intéressé au risque technologique mineur à Strasbourg et a évoqué le problème relatif aux installations classées pour la protection de l'environnement. Mujinga (1997) et Mwanji (2014) ont soulevé la problématique de la répartition spatiale des stations-service à Lubumbashi et leur impact sur l'environnement, thème qui est approfondi dans cette recherche.

De nos jours, les stations-service sont comptées parmi les infrastructures urbanistiques et économiques les plus en vue. Les postes de distribution des produits pétroliers prennent de l'ampleur, non seulement par l'ascension de l'automobile à Lubumbashi mais aussi par l'utilisation, dans la ville, de groupes électrogènes suite aux difficultés d'alimentation en électricité. À cela s'ajoutent les motocyclistes avec leurs taxi-moto qui utilisent une quantité non négligeable de carburant vu leur nombre. Il est à noter que vers les années 1980-1990, l'accès au carburant d'une manière officielle et libre dans la ville de Lubumbashi était difficile, ce qui impliquait la recrudescence du phénomène Kadhafi (vente informelle du carburant dans des bidons). Devant les rares stations qui existaient, on observait des longues files de véhicules attendant d'être servis en carburant. Aujourd'hui, ce phénomène a considérablement diminué avec l'ouverture de plusieurs stations-service et ce, sur différentes artères de la ville. En plus, le prix du 
carburant semble se stabiliser, soit 1,2 dollars par litre depuis 2010, car l'État avait un regard attentif tourné vers le secteur des hydrocarbures.

\section{Méthodes Et Techniques \\ Méthodes}

Cette recherche a fait recours à la méthode descriptive-explicative, dans ce sens que non seulement les secteurs d'étude (communes) ont été décrits dans leur nature, mais aussi les facteurs de localisation des stationsservice ont trouvé des explications dans leur contexte spatial.

\section{Techniques}

La méthode utilisée a fait appel à plusieurs techniques :

- La technique documentaire a consisté en la revue de la littérature sur la thématique en étude. Il s'est dégagé que la question intéresse de plus en plus les géographes en général et ceux de la ville de Lubumbashi.

- L'observation directe a précédé le travail de repérage des stationsservice à l'aide d'un Système de Positionnement Global (GPS), travail qui a été couplé des entretiens libres avec les acteurs impliqués dans cette activité.

- L'enquête par questionnaire élargie aux responsables des stationsservice, aux travailleurs, aux clients et aux agents du Ministère des Hydrocarbures a permis de compléter les informations sur les caractéristiques des stations-service et les risques liés au phénomène.

- Les indices relatifs ou pourcentages calculés à partir du nombre de stations-service par commune rapporté chaque fois au total des stations de la ville ont permis la comparaison du phénomène entre les communes.

- Pour comprendre l'évolution du phénomène dans le temps, il a été fait référence aux données de l'année 1997.

- Le modèle de distribution spatiale des stations- services dans la ville de Lubumbashi a été trouvé à l'aide de la méthode des quadrats.

\section{Présentation De La Ville De Lubumbashi}

La ville de Lubumbashi est consécutive à la découverte de la mine de cuivre à Kalukuluku dans le secteur Est (Mine de l'Étoile). Créée en 1910 par le pouvoir colonial belge, la ville est située à $11^{\circ} 39^{\prime}$ ' $57^{\prime}$ de latitude Sud et $27^{\circ} 28^{\prime}$ ' $35^{\prime}$ ' de longitude Est, à $30 \mathrm{~km}$ de la frontière zambienne au Sud-Ouest. Elle est le Chef-lieu de la province du Haut Katanga et vient au second rang dans la hiérarchie des villes de la République Démocratique du Congo après Kinshasa la capitale. La carte ci-dessous (Figure 1) présente Lubumbashi dans 
sa subdivision administrative. Sur le plan administratif, la ville de Lubumbashi est subdivisée en sept communes. Tel que le montre la Figure 1 ci-dessous.

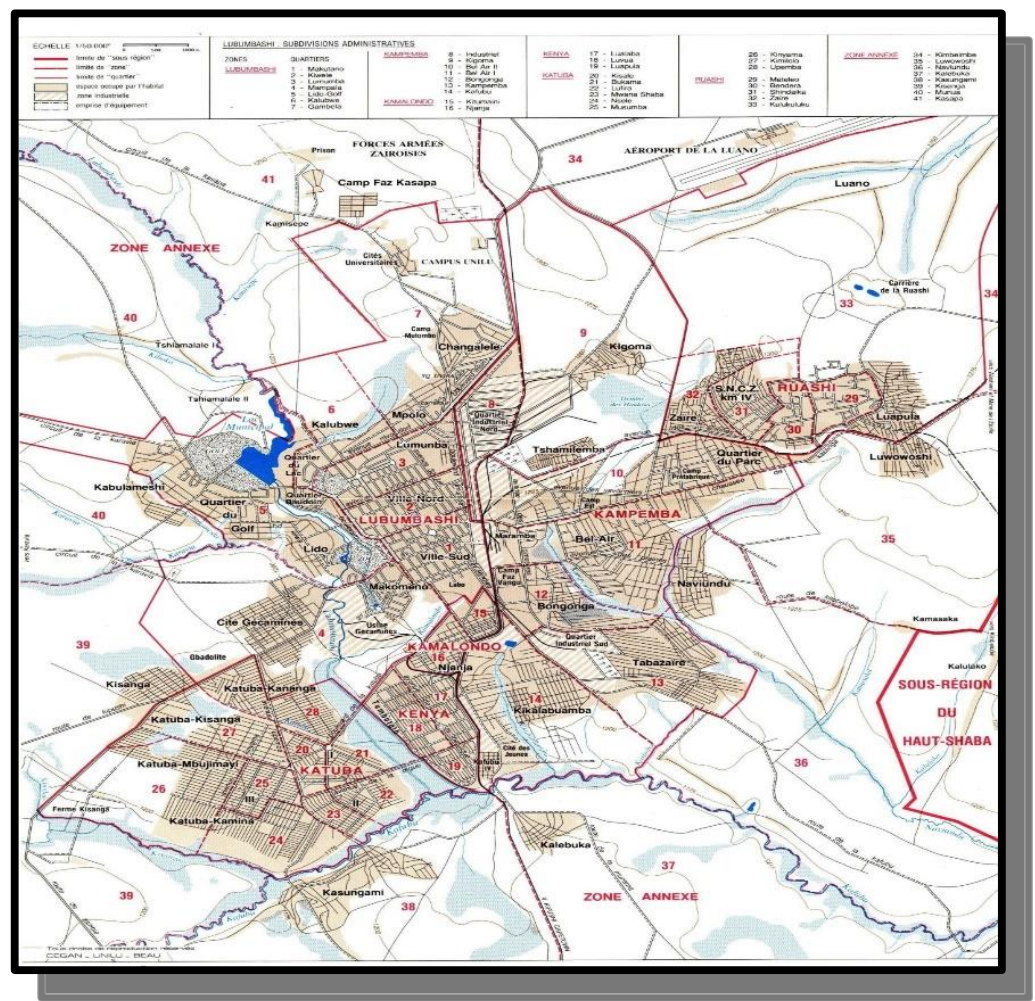

Figure 1. Subdivision administrative de la ville de Lubumbashi

La commune de Lubumbashi, conçue sur le modèle européen, est le symbole de la vie moderne. Les communes de Kamalondo, Kenya, Katuba et de Rwashi ont été créées au départ pour abriter la population noire indigène. Dans la typologie de Bruneau et Le Pain (1990), elles sont qualifiées de Quartiers anciens populaires planifiés. Aujourd'hui, à part les communes de Kamalondo et de Kenya qui n'ont pas la possibilité de s'étendre au-delà de leurs limites anciennes, celles de Katuba et de Rwashi se sont étalées en autopromotion. La commune de Kampemba à l'origine était la banlieue de la ville européenne, puis s'est aussi étendue spatialement en autopromotion. La commune Annexe est constituée de quartiers très dispersés qui auréolent la ville. C'est en fait la ceinture verte de la ville transformée en espaces bâtis.

Du point de vue démo-spatial, le Tableau ${ }^{\circ} 1$ ci-dessous montre que c'est la commune de Kampemba qui est la plus peuplée avec ses 620735 habitants ; elle est suivie de la commune Annexe qui abrite 398274 âmes. 
Tableau 1. Les Communes de la ville de Lubumbashi et leurs populations

\begin{tabular}{|l|c|c|c|}
\hline Commune & Nombre d'Habitants & Superficie $\left(\mathrm{en} \mathrm{Km}^{2}\right)$ & Densité \\
\hline Annexe & 398274 & 640,00 & 622,30 \\
\hline Kamalondo & 36837 & 1,35 & 27286,70 \\
\hline Kampemba & 620735 & 31,50 & 19705,90 \\
\hline Katuba & 322611 & 16,60 & 19434,40 \\
\hline Kenya & 112207 & 2,53 & 44350,60 \\
\hline Lubumbashi & 323062 & 38,00 & 8501,60 \\
\hline Ruashi & 224472 & 16,60 & 13522,40 \\
\hline Total & 2038198 & 746,58 & 2730,00 \\
\hline
\end{tabular}

Source : Mairie de la ville de Lubumbashi

Les communes de Lubumbashi et de Katuba se rapprochent en terme de populations, respectivement 323062 et 322611 habitants, tandis que celles de Kenya et de Kamalondo ont chacune 112207 et 36837 habitants. Quant à leurs superficies, la commune Annexe vient en tête avec $640 \mathrm{Km}^{2}$. On comprend bien qu'elle est périphérique, s'apprêtant aux extensions sous plusieurs formes. Les communes de Lubumbashi et de Kampemba viennent en deuxième et troisième position avec 38 et $31,5 \mathrm{~km}^{2}$. Les communes de Kenya et de Kamalondo sont les moins étendues, elles ont respectivement 2,53 et 1,35 Km², d'où leurs densités très élevées (44350,6 et 27286,7 hab. $/ \mathrm{km}^{2}$ ). Les communes de Kampemba et de Katuba ont des densités proches (19705,9 et 19434,4 hab. $\left./ \mathrm{km}^{2}\right)$.

\section{Présentation Des Résultats}

Il y a lieu de retenir qu'il s'observe un engouement dans l'activité liée à la vente du carburant ces dernières années. Le secteur en question semble rentable pour les opérateurs économiques qui s'y sont intéressés. C'est pourquoi les stations-service se multiplient et celles en gestation ont été réactivées.

Dans la ville de Lubumbashi, 63 Stations-service ont été dénombrées comme le montre le Tableau $\mathrm{n}^{\circ} 2$ ci-dessous, alors que par-ci par-là on s'aperçoit que les terrains sont en train d'être apprêtés pour en recevoir d'autres. 
Tableau 2. Distribution spatiale des stations-service par entité administrative

\begin{tabular}{|l|l|l|l|}
\hline $\mathrm{N}^{\circ}$ & Commune & Nombre de stations-service & Pourcentage \\
\hline 1 & Annexe & 7 & 11,1 \\
\hline 2 & Kamalondo & 1 & 1,5 \\
\hline 3 & Kampemba & 14 & 22,2 \\
\hline 4 & Katuba & 4 & 6,4 \\
\hline 5 & Kenya & 4 & 6,4 \\
\hline 6 & Lubumbashi & 30 & 47,6 \\
\hline 7 & Ruashi & 3 & 4,8 \\
\hline & Total & 63 & 100 \\
\hline
\end{tabular}

On constate que près de la moitié des stations-service (30 sur 63) sont situées dans la commune de Lubumbashi, soit 47,6\%, suivi de la commune de Kampemba avec 14, soit 22,2\%. La commune Annexe compte 7 stationsservice, soit $11,1 \%$. Les communes de Katuba et de Kenya ont chacune 4 stations-service, soit 6,4 \% et celle de Ruashi 3 stations-service soit 4,8 \%. La commune de Kamalondo n'en a compté qu'une seule, soit 1,5\%.

En effet, la commune de Lubumbashi jouit d'une centralité forte à cause de nombreuses activités qui s'y exercent. Elle est le cadre du CBD (Center Business District) : commerce de gros et de détail, transactions bancaires, mais aussi elle est le cadre des institutions politico-administratives : gouvernorat, palais de justice, divisions administratives, police et justice militaire et les grandes institutions sanitaires et éducatives. Ces activités font qu'au quotidien, les flux des personnes convergent vers le centre-ville et viennent s'ajouter aux habitants de la commune. Un autre facteur qui favorise l'implantation des stations-service dans la commune de Lubumbashi c'est l'aspect sécuritaire, car le périmètre de cette commune semble bien être surveillé le jour comme la nuit, rassurant les tenanciers des stations-service par rapport aux cambrioleurs et aux risques d'incendies.

La commune Annexe est très éclatée en plusieurs quartiers occupant la périphérie de la ville. Elle accueille des stations qui vont à la rencontre des usagers lointains ou qui ont du mal à se placer au centre-ville à cause du prix élevé des terrains. L'information obtenue des tenanciers des agences immobilières révèle que le prix le plus bas d'une parcelle dans le secteur central de la ville se situe actuellement autour d'un million de dollars, autour de quatre à deux cents mille dans la zone médiane, jusqu'à dix mille dollars, voire moins, en périphérie de la ville. En réalité, la spéculation foncière est de mise. Cela dépend aussi de la situation économique du moment.

Les communes de Katuba et de Kampemba sont vastes et peuplées et commencent à être convoitées par les opérateurs pétroliers. Par contre, les communes de Kenya et de Kamalondo sont trop étroites (respectivement 2,53 
et $1,35 \mathrm{Km}^{2}$ ) et coincées dans leurs limites ; pour la première, elle n'a que deux grandes artères où passent les véhicules de transport en commun et ces deux artères se trouvent dans la longueur de la commune, il s'agit des avenues Basilique et Du Marché. Quant à la seconde, elle n'a pas de terminus de transport en commun. À part quelques rares taxis qui y passent pour récupérer les gens qui vont au centre-ville ou dans d'autres communes. Elle manque d'espaces qui peuvent intéresser les pétroliers pour y implanter des stationsservice.

Si l'on compare avec les données obtenues par Mujinga (1997), on peut constater que les stations-service ont augmenté sensiblement. Sur les 44 stations dénombrées par l'auteur, 23 seulement, soit 52,3\%, étaient en activité et 21 autres, soit $47,7 \%$ en dysfonctionnement. Considérant uniquement les 23 stations opérationnelles en 1997 et les 63 en 2015 révélées par l'enquête de cette étude, il se dégage que 40 stations-service se sont ajoutées, soit un accroissement de $174 \%$. Ce fait serait dû au changement de politique du pays en matière des hydrocarbures, notamment la libéralisation du commerce de pétrole, l'amélioration des conditions d'importation et surtout la subvention de l'État. Il faudra noter que l'État congolais a un regard très particulier sur ce secteur qui a des multiples implications dans la vie nationale.

\section{Organisation Spatiale Des Stations-Service À Lubumbashi}

Lorsqu'on considère la ville de Lubumbashi dans son ensemble, les stations-service apparaissent de la manière illustrée par la Figure $\mathrm{n}^{\circ} 2$ cidessous.

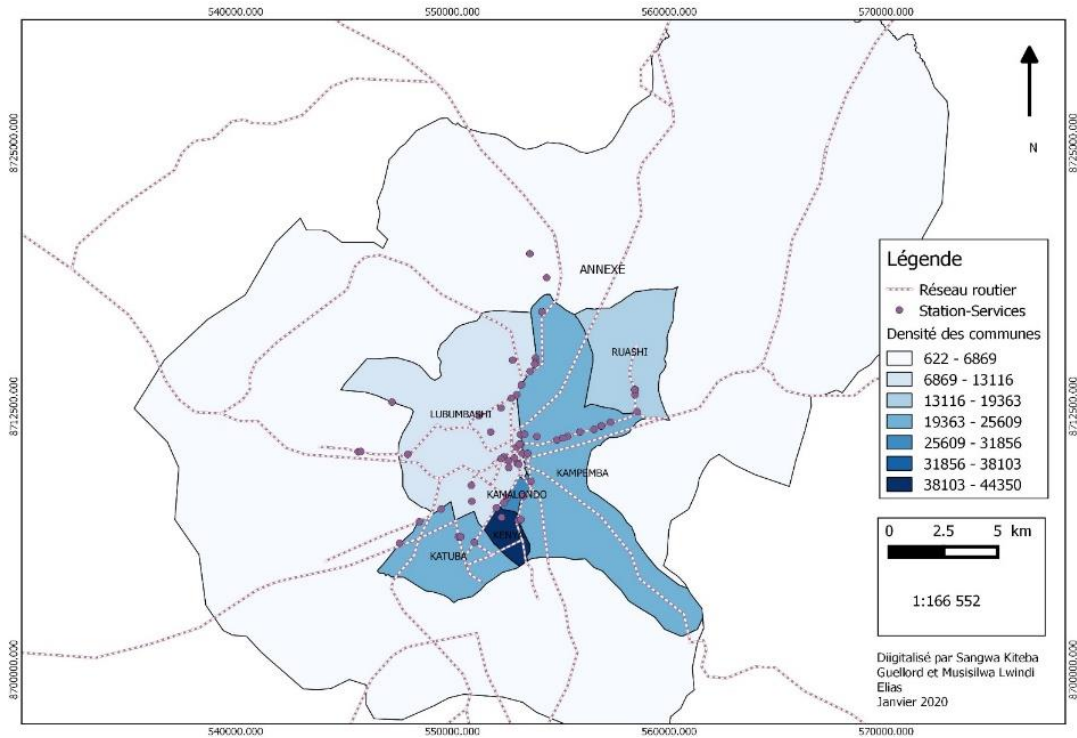

Figure 2. Carte de la répartition spatiale des stations-service et de la densité de la population 
Trois tendances se dégagent. Premièrement, leur forte concentration est observée au niveau du secteur central qui est, comme cela a été mentionné précédemment, le domaine du CBD. Deuxièmement, dans le secteur sudouest, les services de l'offre du carburant sont très dispersés. Ce secteur concerne la commune Kenya et Kamalondo, les plus densément peuplées et qui n'offrent pas suffisamment de possibilités pour la localisation des stationsservice, et la commune Katuba qui n'attire pas beaucoup les opérateurs pétroliers. Troisièmement, le phénomène étudié a une distribution quasi linéaire sur trois directions : Route Kasenga, Route Likasi et Route Kipushi (Kasumbalesa).

Il a été fait recours à la méthode des quadrats pour trouver le modèle de distribution spatiale des stations-service à Lubumbashi.

En effet, le quadrillage de l'espace occupé par les points qui représentent les stations-service (Figure 3) calqué sur la carte réalisée (Figure 2) a permis de dresser le tableau $n^{\circ} 3$ de données couplées (nombre de cases $X$ nombre de stations-service).

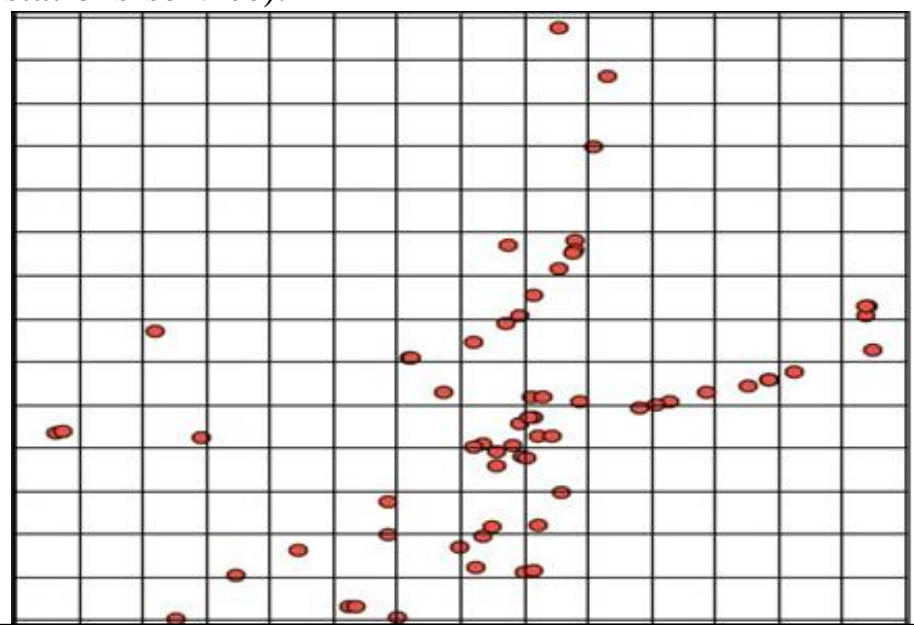

Figure 3. Localisation de points selon la méthode des quadrats

Tableau 3. Correspondance nombre de cases-nombre de stations-service Données couplées (nombre de cases x nombre de stations-service).

\begin{tabular}{|l|l|}
\hline Nombre des cases & Nombre des stations par cases \\
\hline 170 & $\mathrm{O}$ \\
18 & 1 \\
7 & 3 \\
6 & 2 \\
3 & 4 \\
\hline
\end{tabular}

Nombre de points : 63

Nombre de cases : 204

Le processus de traitement de ces données et le résultat apparaissent ci-dessous.

$\bar{x}=63 \div 204=0,3088$ 


$$
\begin{aligned}
& S^{2}=\frac{170(0-0,308)^{2}+18(1-0,308)^{2}+7(3-0,308)^{2}+6(2-0,308)^{2}+3(4-0,308)^{2}}{204-1} \\
& S^{2}=0,6578 \\
& \frac{S^{2}}{\bar{x}}=2,1357
\end{aligned}
$$

Comme 2,1357 est supérieur à 1, la distribution des stations-service à Lubumbashi est du type concentré avec agrégats. On peut tester la significativité de cette hypothèse (d'une répartition concentrée avec agrégats) au moyen du test $\mathrm{z}$ (puisque $\mathrm{m}$ est grand et vaut 204, et la moyenne inférieure à 5).

$$
Z=\frac{\llbracket(m-1) \div 2 \rrbracket^{\frac{1}{2}}}{1} \cdot \frac{S^{2}}{\underline{X}}-1=\frac{\llbracket(204-1) \div 2 \rrbracket^{\frac{1}{2}}}{1} \cdot \frac{0,6578}{0,308}-1=11,4418
$$

La valeur du test n'étant pas comprise dans l'intervalle de confiance ($1,96 ; 1,96)$ pour une distribution de probabilité normale centrée réduite avec alpha $=5 \% ; 1$ 'hypothèse est donc vérifiée. Le modèle en question illustre une forte prédominance des stations dans le secteur qui correspond au CBD de la ville avec tout ce qui a été décrit comme activité ; il se profile en deux axes, vers le Nord et vers l'Est, lesquelles sont en opposition avec celles disséminées au Sud et à l'Ouest.

Compte tenu de cette répartition, il y a lieu d'avancer que les activités intenses et les grandes artères sont des facteurs favorisant l'implantation des stations-service à Lubumbashi.

\section{Le Point De Vue Des Tenanciers Des Stations-Service Concernant Leur Localisation}

Une enquête a été menée auprès des tenanciers ou responsables des stations-service. Unanimement, ils ont soutenu que l'augmentation du nombre de charroi automobile suscite la demande en produits pétroliers ; et qu'il faut exercer des activités qui rapportent de l'argent, étant la vente du carburant une d'entre elles. Les motifs de placement des stations-service sont les suivants :

- Pour $21,1 \%$ des enquêtés, il faut se mettre sur une voie accessible, comme par exemple la Route Likasi, la Route Kasenga et la Route Kipushi. Ces voies enregistrent des flux importants de véhicules.

- Il est question, pour 17,3\% des personnes interrogées, de trouver un endroit stratégique; c'est-à-dire, où l'on a la possibilité d'avoir beaucoup de clients. C'est pourquoi beaucoup de stations sont placées aux carrefours ou aux angles des avenues.

- Certains tenanciers des stations ont cherché des endroits où celles-ci ne sont pas nombreuses pour éviter la concurrence. C'est le cas de 13,5\% de l'ensemble touché par l'enquête. 
- Pour $48,1 \%$ des sujets interrogés, les motivations sont variées, entre autres : la sécurité, la disponibilité offerte par une ancienne station déjà suffisamment équipée, etc.

\section{Attractivité Des Stations-Service}

D'après les informations recueillies sur terrain, les causes qui attirent les clients de produits pétroliers sont liées au prix abordable (pour 33, 3\% des enquêtés) ; à la qualité du produit pétrolier pour $25 \%$; à la proximité routière pour $16,7 \%$; à la rapidité dans la livraison de produits $(13,3 \%)$ contre $11,7 \%$ des personnes interrogées qui sont attirées par l'accueil des agents commis à cette activité.

\section{Risques Lies Aux Stations Services En Milieu Urbain}

Il est important de parler tout d'abord des impacts environnementaux par rapport à leur origine et leurs effets sur les différents compartiments environnementaux (l'air, l'eau, le sol, ...). Ensuite, les mesures de prévention de pollution seront évoquées sous forme de recommandations pratiques.

\section{Impact Sur L'eau}

\section{Sources de polluants}

Lors $\mathrm{du}$ fonctionnement normal d'une station-service, l'eau est souvent utilisée comme moyen d'évacuation de divers polluants. Les eaux de pluie, de robinet, des canalisations et des cours d'eau lessivant des surfaces sont chargées d'impuretés comme des particules solides ou des différents types de substances utilisées par les véhicules, notamment les hydrocarbures, les lubrifiants ou les détergents. À cela s'ajoutent des déversements accidentels d'autres liquides classés comme des substances dangereuses, couramment présents dans les stations-service (p.ex. gasoil, essence, huile de freins, liquides de refroidissement, acides de batteries ou solvants) et des fuites de réservoirs souterrains.

\section{Effets Des Polluants}

Si les eaux usées résultant des activités de la station-service étaient déversées sans traitement dans la nature, les substances y contenues auraient, pour la plupart, des effets toxiques sur les écosystèmes aquatiques et végétales. La tuyauterie en contact avec les éléments toxiques subirait la corrosion. Les hydrocarbures (essence, gasoil, etc.) par exemple se décomposent sous l'action de bactéries en molécules plus légères (benzène, toluène, Xylène) très toxiques et solubles dans l'eau. Les huiles à moteur (usagés ou non) contiennent également des substances toxiques (par effets additifs), souvent difficilement dégradables. Les huiles forment par ailleurs un film à la surface de l'eau qui empêche les échanges gazeux. Les détergents 
contiennent des agents tensioactifs qui favorisent la formation de mousse, dissolvent la graisse et peuvent être difficilement dégradables, des propriétés qui ont des effets très néfastes sur les écosystèmes aquatiques comme sur le fonctionnement des stations d'épuration (Van, 1990).

Cela étant, quelques exemples des effets possibles ont été choisis et présentés ci-après. Les liquides de freins étant souvent des mélanges de dérivés glycoliques, ont des propriétés toxiques pour de nombreux organismes aquatiques à partir de certaines concentrations. De plus, leur dégradation par des micro-organismes mène à une consommation d'oxygène dans l'eau. De plus, les liquides de frein usagés peuvent contenir des impuretés comme des métaux lourds, qui peuvent être toxiques aux organismes aquatiques et à l'homme, même à très petite dose. Les liquides de refroidissement sont souvent des mélanges d'eau distillée (environ 60\%) et de glycol (environ 40\%), agissant comme antigel et inhibiteur de corrosion (Van, 1990).

Les batteries des voitures contiennent du plomb et de l'acide sulfurique. Le plomb est un métal lourd qui a tendance à s'accumuler dans les chaînes alimentaires en provoquant des intoxications graves (Van, 1990).

L'acide sulfurique est une substance corrosive et également toxique pour la vie aquatique. Les solvants ont aussi souvent des propriétés toxiques pour la vie aquatique et sont difficiles à éliminer, même par une station d'épuration. Les huiles de graissage diminuent les échanges gazeux air/eau, s'accumulent et créent des dépôts pouvant aller jusqu'au dysfonctionnement et à l'obstruction des systèmes d'assainissement (Van, 1990).

Les hydrocarbures, toxiques pour les organismes vivants supérieurs, peuvent lors d'un déversement accidentel, entraîner la contamination des sols, des nappes phréatiques et des cours d'eau, détruisant les écosystèmes en place et interdisant l'utilisation des ressources en eau pour de nombreuses générations (Van, 1990).

\section{Impacts Sur Le Sol Sources De Polluants}

Dans le domaine de l'exploitation des stations-service, les principales sources de pollution sont liées à des déversements accidentels (p.ex. égouttages lors du remplissage des réservoirs et du ravitaillement des voitures, fuites observées sur les tuyauteries, fuites sur les réservoirs, accidents ou incendies de véhicules, manipulation de produits et lors d'évacuation de déchets, etc.) de produits nuisibles pour l'environnement (carburants, huiles de moteur, liquides de frein, liquides de refroidissement, acides de batteries, etc.).

Il y a lieu d'ajouter qu'à Lubumbashi et partout d'ailleurs où l'on trouve des stations-service en RD Congo, tous les lieux occupés sont pavés. Deux faits peuvent facilement être attendus, la réflexion de la chaleur absorbée 
avec augmentation de la température ambiante et des forts ruissellements des eaux de pluies exacerbant les inondations, très courantes ces dernières années dans la ville.

\section{Effets Sur La Santé}

Un polluant dans le sol est dangereux s'il devient mobile et s'il atteint sa cible (eaux souterraines ou superficielles, écosystème, ...). Un sol pollué devient alors un problème de santé publique si le polluant atteint la nappe phréatique ou le cours d'eau avoisinant. Dans le quartier Kalubwe, à l'Ouest de la ville de Lubumbashi, Sompo (2019) a mis en évidence la contamination des eaux de puits proches des stations dans ce secteur. Les eaux que l'on puisait dégageaient l'odeur des hydrocarbures.

Avant de faire un examen du lien entre les effets des polluants des sols provenant des stations-service et la santé, il a été tenu compte de quelques considérations d'ordre général. Les facteurs de risques pour la santé sont multiples. Des facteurs génétiques ou comportementaux interviennent aussi.

Il existe un certain nombre d'étapes entre le milieu extérieur et les effets observés sur l'homme :

- Absorption et distribution dans les différents compartiments de l'organisme avec éventuellement

- Bioaccumulation et effets à court, voire à long terme.

L'absorption digestive est très différente selon que l'on a affaire à un enfant ou un adulte. Beaucoup de facteurs peuvent favoriser l'absorption : la teneur alimentaire en acides organiques, les carences en fer, ou en fibres végétales, l'état de jeûne, même chez l'adulte.

Par ailleurs, le risque lié à une exposition et la mesure de l'impact des polluants sur la santé va dépendre des éléments suivants :

D'abord, la relation entre l'exposition à un cocktail d'agents n'est pas une relation simple. La toxicité d'une molécule peut être augmentée ou diminuée par l'exposition simultanée ou consécutive à une autre molécule (Van, 1990). On dit qu'il peut y avoir :

- $\quad$ synergie : les effets sont dus à l'action de plusieurs molécules qui, lorsque prises isolément, n'ont que peu d'action ;

- antagonisme : l'effet d'une molécule s'oppose à celle d'une autre ;

- cumul: les effets d'une molécule accélèrent les effets d'une ou plusieurs autres molécules.

Ensuite, les effets directs sur la santé peuvent être constatés auprès de la population proche d'un site qui subit une pollution du sol par consommation d'aliments produits sur place, mais dans le cas des stations-service, c'est peu 
probable que cela arrive étant donné que ce type d'installations est en principe éloigné des zones agricoles ou des jardins potagers.

Les enfants figurent parmi les populations les plus vulnérables et ce de par leur grande « sensibilité biologique », mais également à cause de certains comportements (ingestion de terres contaminées, contact plus fréquent avec le sol, etc.).

L'exposition indirecte concerne par exemple l'ingestion des eaux contaminées à travers les nappes phréatiques. Cela ne préjuge en rien l'exposition effective à un polluant, laquelle intègre la notion de durée et de proximité de la source d'émission par rapport à la personne.

Si sur le terrain une station-service reste en exploitation et que la pollution reste limitée au terrain lui-même, il n'y aura pas de risque actuel pour la santé publique dans de nombreux cas (Bofas, 2004).

Suivant la classification de l'Union Européenne (Van, 1990), les risques de l'essence automobile pour la santé sont le cancer et une atteinte des poumons en cas d'ingestion.

En ce qui concerne le gazole, un effet cancérogène est suspecté, mais les preuves sont insuffisantes. Il peut provoquer aussi une atteinte des poumons en cas d'ingestion.

Les huiles minérales possédant un point d'ébullition bas provoquent des pneumonies chimiques de gravité importante et d'évolution lente en comparaison avec les hydrocarbures légers. La plupart des liquides de frein sont très nocifs à l'ingestion, notamment le polyéthylène glycol.

\section{Effets Sur L'environnement}

Les risques écologiques sont plutôt de moindre importance à partir des pollutions par les stations-service. En général, une menace des écosystèmes est rarement constatée à proximité d'une station-service étant donné qu'elle est toujours située le long d'une voie publique où la présence d'écosystèmes séparés est limitée. Par ailleurs, étant donné la portée spatiale limitée des stations-service et la présence d'un revêtement sur une grande partie du terrain, l'aspect des risques pour l'écologie est souvent moins pertinent (Bofas, 2004).

Toutefois l'existence des risques pour l'écosystème n'est pas exclue. En cas de déversement ou de fuites dans le milieu naturel, selon les circonstances, les fractions non volatiles se disperseront dans l'environnement aquatique ou seront absorbées dans le sol créant éventuellement une pollution des nappes souterraines pouvant induire une contamination des zones de captage d'eau potable.

Dans l'eau, les carburants ont tendance à flotter et à s'étendre à la surface à cause de la faible solubilité de certains de leurs constituants formant une nappe « huileuse » qui empêche les échanges gazeux entre l'air et le milieu 
aquatique. Ceci entraîne à terme une sous-oxygénation et peut provoquer la mortalité de la faune aquatique (Centre Canadien d'Hygiène et de Sécurité au travail, 2007).

\section{Exposition De L'habitat Lushois Aux Risques Liés Aux Stations-Service}

La prolifération des stations-service dans la ville de Lubumbashi présente un danger imminent.

Premièrement, les stations commencent à conquérir l'habitat. En dehors des stations traditionnelles, les nouvelles stations n'ont pas de zone tampon par rapport à l'habitat; ce qui veut dire que le jour où il y aura un incendie, la chance de déplorer beaucoup des morts est grande.

Deuxièmement, beaucoup de stations sont proches l'une de l'autre. C'est le cas, par exemple, de deux stations : celle au croisement des Avenues des Usines et Lomami et celle au croisement des Avenues Lomami et Likasi. Deux stations près du Camp Est (camp de police) sur l'Avenue Chaussée de Kasenga seront aussi citées.

Il suffit qu'une station prenne feu pour que tout un ilot de la ville soit embrasé ; on aura des explosions en série. Il y a deux ans, un dépôt de carburant avait pris feu sur l'Avenue des Cimetières occasionnant mort d'hommes et des dégâts matériels importants.

Troisièmement, ces stations-service n'ont pas de garantie de sécurité si bien que le service anti incendie est positionné loin de ces activités, à l'aéroport de la Luano distant par exemple du centre- ville d'à peu près $15 \mathrm{~km}$ ou à la Mairie quoique proche de beaucoup de stations ; le secours est toujours tardif, les engins n'étant pas toujours apprêtés pour intervenir à temps.

À cet égard, l'État devrait limiter le nombre de stations-service, réguler leur répartition à travers la ville et rendre rigoureuses les conditions d'acquisition de permis d'ouverture des activités de vente du carburant.

\section{Conclusion}

L'analyse diachronique des stations-service révèle que ces dernières connaissent un accroissement rapide en nombre ; de 44 en 1997 dont 19 en activité, à 63 en 2015, ce qui montre que les stations-service sont en plein essor dans la ville de Lubumbashi. Leur répartition rappel un modèle spatial de type concentré avec agrégat. Cette concentration est observée dans les lieux où les activités urbaines sont intenses : centre-ville ; carrefours et le long des grands axes.

L'étude a également évoqué les risques des stations-service dans la ville, notamment les probables pollutions qui ont des effets sur la santé des habitants en particulier et sur l'écosystème urbain en général jusqu'à stigmatiser le danger de ces stations-service par rapport à leur rapprochement, à cause de la négligence du service de régulation qui tolère leur construction 
dans la population sans tenir compte de la zone tampon, alors que les mesures de protection contre les incendies sont inefficaces.

L'État devrait avoir un regard attentif sur le développement du phénomène et réglementer l'ouverture des stations-service, si possible, délocaliser plusieurs d'entre elles afin de réduire le risque d'incendie mortel.

\section{References:}

1. Blaesius, J. (1992). «Stations hors service », Dernières nouvelles d'Alsace, 6 décembre, p. 4.

2. Bofas (2004). Directive sur l'assainissement des sols des stationsservice en Belgique. Bruneau JC. et le Pain M., (1990). Atlas: Atlas de Lubumbashi. Centre d'Etudes Géographiques sur l'Afrique noire et Université de Paris X, Nanterre, 133p.

3. Calvat, J.M. (1988). « Les stations-service disparaissent », Inventaire communal de 1988, pp. 23-26.

4. Cauvin, C. \& Rimbert, S. (1976). Les méthodes de la cartographie thématique. Fascicule: Lecture numérique des cartes thématiques, Éd. Université de Fribourg, Suisse, 172 p.

5. Dupuy, G. (1995). Les Territoires de l'automobile, Paris, Anthropos Economica, 216 p. Fabrice De Monicault., (2013). «Une activité très disputée », in Le Figaro, encart «Économie », jeudi 21 novembre.

6. Hiegel, C. (1995). Au risque de la ville... Les stations-service à Strasbourg. Un risque technologique mineur. Mémoire de DEA, UFR Géographie Strasbourg, 126 p.

7. Mujinga, K. (1997) et Mwanji (2014). Répartition des stations-service dans l'espace urbain' 'cas de la ville de Lubumbashi '. Mémoire de fin d'étude au Département de Géographie, Université de Lubumbashi) ,64p.

8. Nordemann, F. (1981). \#Bâtiments de l'automobile, Paris, Seuil, 59 p.

9. Sompo, K. (2019). Qualité Physico chimique et organoleptique des eaux de puits et de forages aux environs des stations-service 'Cas des stations-service Bon Berger et Groupe Petro Peuple au quartier Kalubwe'". Mémoire de fin d'étude, Département de Géographie, Université de Lubumbashi. Travail de fin Bachelier.65P.

10. Van, W. (1990). La mesure de l'impact des polluants sur la santé, Belgique. 